\title{
Lambda Plate Seal for HFVMTF Anticryostat
}

\author{
Brett Gasior, University of Kentucky - SULI Program | Sergey Koshelev - Fermilab
}

\section{Introduction}

Fermilab is currently designing a High Field Vertical Magnet Test Facility in order to test superconducting cables and magnets. The goal of this project was to analyze the thermal performance of three different seal designs and provide recommendations for the HFVMTF. Seal Designs
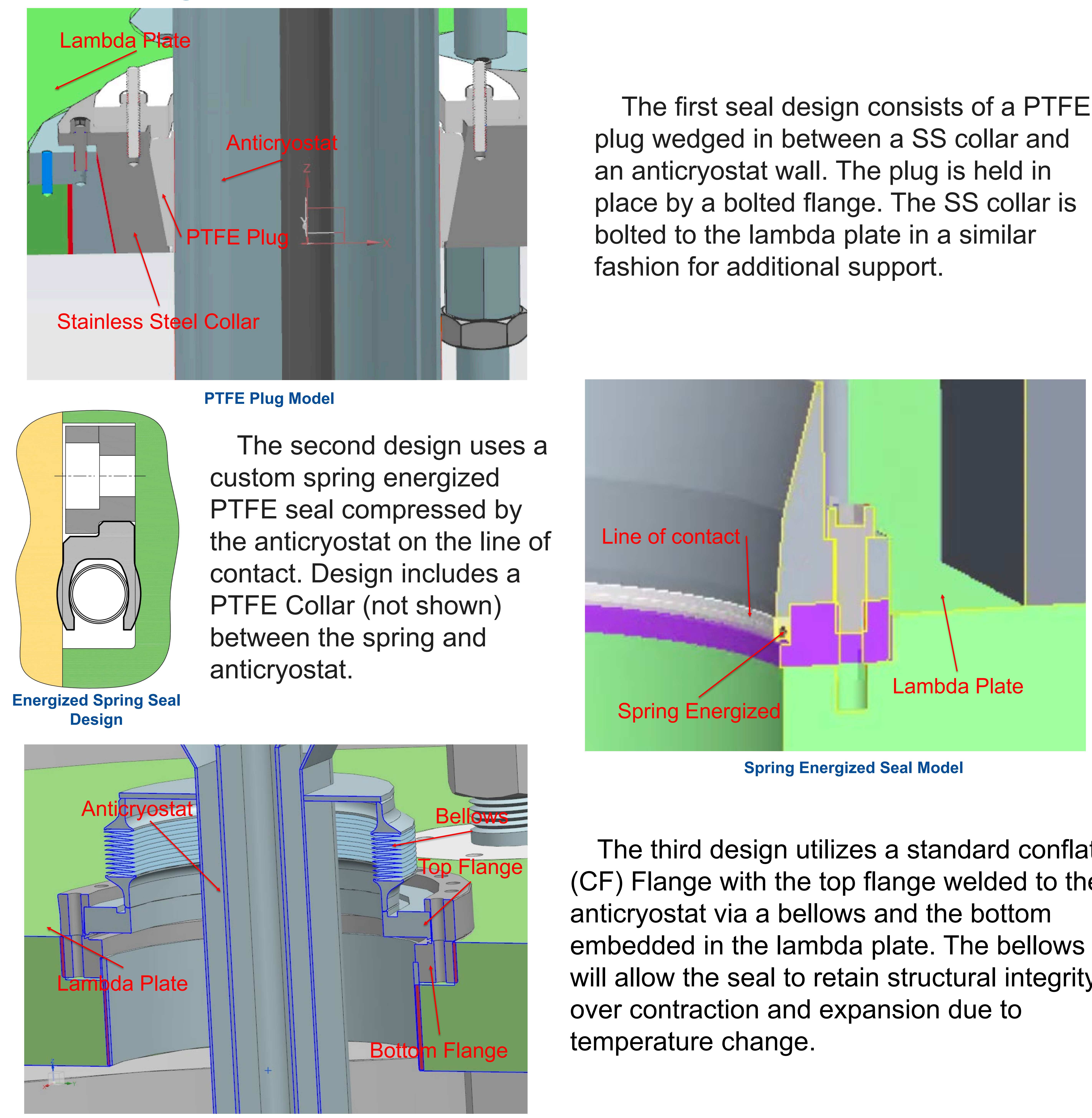

The third design utilizes a standard conflat (CF) Flange with the top flange welded to the anticryostat via a bellows and the bottom embedded in the lambda plate. The bellows will allow the seal to retain structural integrity over contraction and expansion due to temperature change.

\section{Analysis}

Preliminary analysis on simplified geometry provided us with a baseline for the heat flow rate and showed that the CF

Flange design needed improvement. This design was updated by using vacuum insulation instead of a single wall bellows to reduce heat flow. The seals were modeled in more detail and finite element analysis was performed to estimate the heat flow rate. Structural analysis of the updated CF Flange Seal was performed for design verification.
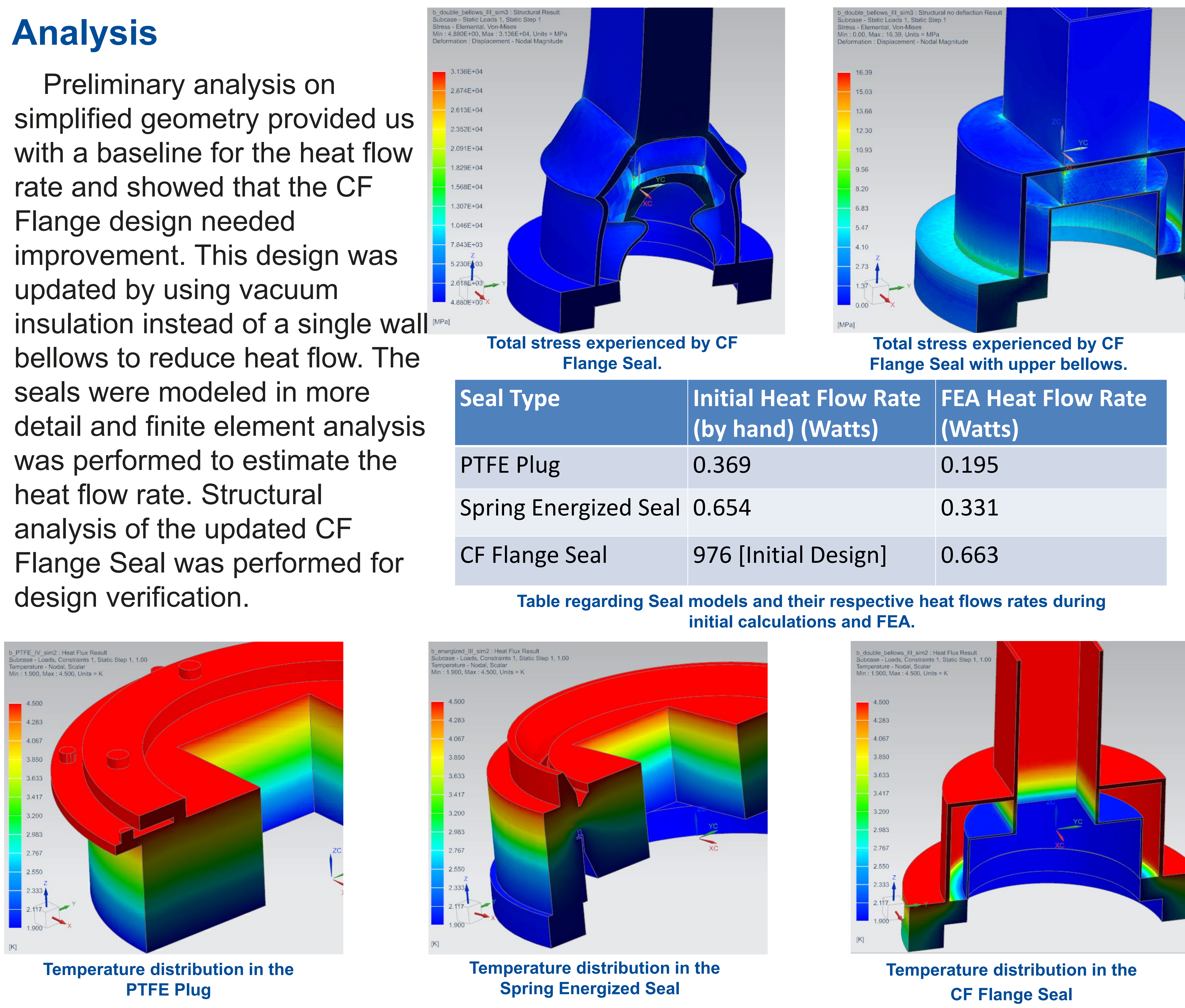

\begin{tabular}{lll} 
Seal Type & $\begin{array}{l}\text { Initial Heat Flow Rate } \\
\text { (by hand) (Watts) }\end{array}$ & $\begin{array}{l}\text { FEA Heat Flow Rate } \\
\text { (Watts) }\end{array}$ \\
\hline PTFE Plug & 0.369 & 0.195 \\
\hline Spring Energized Seal & 0.654 & 0.331 \\
CF Flange Seal & 976 [Initial Design] & 0.663 \\
\hline \multicolumn{2}{r}{ Table regarding Seal models and their respective heat flows rates during } \\
initial calculations and FEA.
\end{tabular}
initial calculations and FEA.
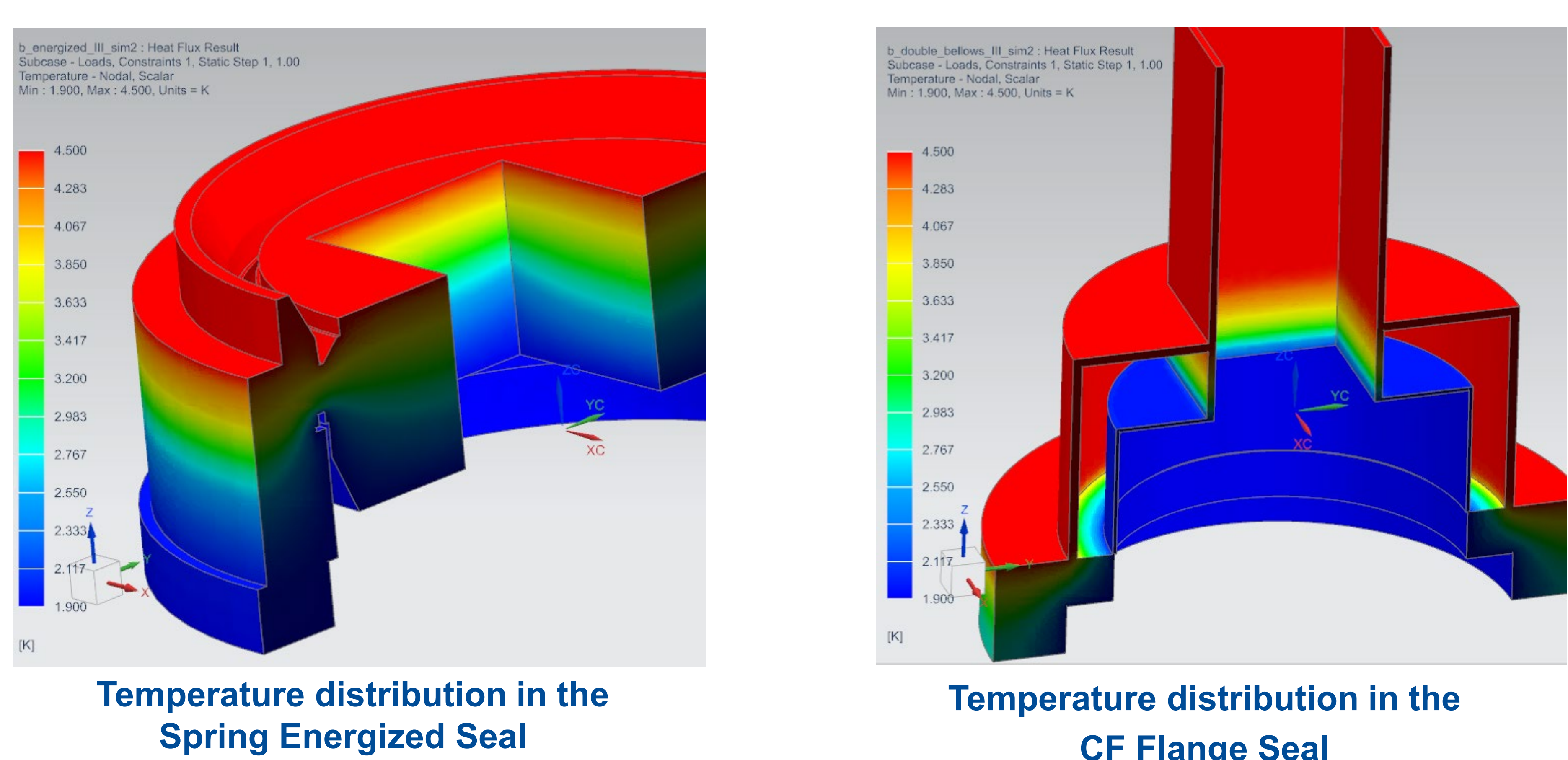

\section{Results and Conclusion}

The PTFE Plug showed the best thermal performance. It has a quality track record of use at Fermilab. The Spring Energized Seal has a slightly higher heat flow rate and has been used at BNL successfully. The CF Flange has the worst thermal performance of three and requires a bellows on the anticryostat due to the excessive stresses it undergoes caused by thermal contraction. The effect of superfluid helium leaks on the heat flow wasn't included in the analysis. This introduced bias against the CF flange seal, having potentially higher leak tightness. Investigation of heat flow rate due to superfluid helium is recommended. Final recommendation for the HFVMTF at this moment is the PTFE Plug. 


\section{Abstract FERMILAB-POSTER-21-098-STUDENT}

Fermilab is currently designing a High Field Cable Test Facility. Its purpose is to test superconducting cables and magnets. The goal of this project was to analyze the thermal performance of three different seal designs and provide recommendations for the HFVMTF. The seals being tested were a PTFE Plug, an Energized Spring Seal and a CF Flange Seal. Primary analysis provided us with a baseline for the heat flow rate. Modifications were made to the CF Flange Seal due to poor performance. The seals were then modeled in more detail and FEA was run to get a more specific grasp of the heat flow rate. Structural analysis was done on the CF Flange Seal due to a possibility of over constraining. The results of the project found that the PTFE Plug would be the most advantageous seal to use in the HFVMTF.

Work supported by the Fermi National Accelerator Laboratory, managed and operated by Fermi Research Alliance, LLC under Contract No. DE-AC02-07CH11359 with the U.S. Department of Energy, Office of Science, Office of High Energy Physics. The U.S. Government retains and the publisher, by accepting the article for publication, acknowledges that the U.S. Government retains a non-exclusive, paid-up, irrevocable, world-wide license to publish or reproduce the published form of this manuscript, or allow others to do so, for U.S. Government purposes. 